\title{
Emergency intubation in trauma in KwaZulu-Natal Province, South Africa
}

\author{
C T Lewis, ${ }^{1} \mathrm{MB}$ ChB; J Brown, ${ }^{2}$ BTech; A C Inglis, ${ }^{3}$ FACEM; D N Naumann, ${ }^{4}$ MRCS; N Crombie,${ }^{4}$ FRCA \\ ${ }^{1}$ Academic Foundation Programme, Raigmore Hospital, Inverness, UK \\ ${ }^{2}$ Netcare 911, Pietermaritzburg, South Africa \\ ${ }^{3}$ Emergency Medicine, Edendale Hospital, Pietermaritzburg, South Africa \\ ${ }^{4}$ National Institute for Health Research, Surgical Reconstruction and Microbiology Research Centre, Birmingham, UK
}

Corresponding author: C T Lewis (christopher.lewis11@nhs.net)

\begin{abstract}
Background. Advanced airway management is a research priority in prehospital care. There is a high burden of major trauma in KwaZuluNatal (KZN) Province, South Africa (SA), and transfer times to trauma units are often prolonged.

Objectives. To examine emergency intubation practice in trauma and burns patients in Pietermaritzburg, KZN, and its environs.

Methods. This was a prospective consecutive case series, conducted from 11 May to 17 July 2016. Data were collected from urban emergency department (ED), rural hospital and roadside procedures in Pietermaritzburg and its drainage area. Patients with emergency intubation following trauma were eligible for inclusion. The primary outcome was successful airway management. Secondary outcomes included first-pass success and adverse events.

Results. Forty-one cases were recorded in patients aged $1-60$ years. No instances of unsuccessful airway management were reported. Recorded first-pass intubation success rates were higher in receiving EDs than rural hospitals (19/22 v. 2/7; $p=0.003)$. Use of a formal preintubation checklist was associated with a higher first-pass success rate $(21 / 23 \mathrm{v} .6 / 15 ; p=0.001)$ and fewer adverse events $(0 / 23 \mathrm{v}$. 7/16; $p<0.001$ ). Identified adverse event rates were 1/22 (EDs), 5/8 (rural hospitals) and 2/9 (roadside). Unmedicated intubation was more common in rural hospitals than EDs ( $3 / 8 \mathrm{v} .1 / 22 ; p=0.019)$, despite absence of cardiac arrest in these cases. Minimum standards of anaesthetic monitoring were not consistently met in any setting.

Conclusions. The use of a preprocedural checklist was associated with improved intubation outcomes and may improve practice in SA trauma care and the prehospital environment, including in rural hospitals. Standardised rapid sequence induction protocols, routine use of introducers and end-tidal carbon dioxide monitoring, and increased availability of intraosseous devices also merit consideration. Key performance indicators should be monitored routinely.
\end{abstract}

S Afr Med J 2018;108(8):660-666. DOI:10.7196/SAMJ.2018.v108i8.12670

Advanced airway management is a research priority in prehospital care. ${ }^{[1]}$ Evidence to support prehospital emergency intubation in trauma is inconclusive, and the skill of the operator may be key in determining efficacy. ${ }^{[2,3]}$ Competence requires considerable training and experience, particularly in the prehospital environment, where conditions are frequently challenging. ${ }^{[4,5]}$

KwaZulu-Natal (KZN) Province is the most populous province in South Africa (SA), and experiences a high burden of trauma. ${ }^{[6]}$ Pietermaritzburg is the region's second-largest city after Durban, and has two hospitals that receive major trauma referrals from peripheral hospitals up to $200 \mathrm{~km}$ away as part of an informal provincial trauma network. ${ }^{[7]}$

Doctors do not routinely work in KZN's prehospital environment, where the need for a cohesive prehospital emergency service has been documented. ${ }^{[7]}$ Transfer times from rural hospitals and incident scenes are often prolonged by geographical challenges and limited services. Paramedics with Advanced Life Support and BTech qualifications are trained to perform advanced airway interventions and transport intubated patients. Advanced Life Support-trained paramedics use a regimen of morphine and midazolam to achieve deep sedation in order to perform tracheal intubation, while those with a BTech degree may undertake rapid-sequence inductions using conventional induction agents and neuromuscular blockade. ${ }^{[8]}$

There is a dearth of data regarding rural hospital and prehospital intubation outcomes in KZN. Clinicians with formal advanced airway training may not be available in the rural setting. ${ }^{[9]}$ The need to improve anaesthetic skills in rural doctors in SA has been identified as a national research priority. ${ }^{[10]}$

\section{Objectives}

To examine emergency intubation practice for trauma and burns patients in the KZN region of SA, and to compare outcomes between emergency department (ED), rural hospital and roadside settings in order to determine whether there is scope for improving patient care.

\section{Methods \\ Study design}

A prospective consecutive case series was undertaken to examine emergency intubation practices in trauma and burns patients in KZN, SA. Prior approval was granted by the University of KwaZulu-Natal Biomedical Research and Ethics Committee (ref. no. BE048/16). The approved protocol included a waiver of informed consent, as patients meeting the inclusion criteria lack capacity.

\section{Study setting}

$\mathrm{KZN}$ is the most populous province in SA, and experiences a high burden of trauma. ${ }^{[6]}$ Prehospital care is currently delivered by both public and private sector providers. Ambulances attend road traffic accidents without discrimination, as providers are financially 
compensated through the government's Road Accident Fund. A Red Cross/Air Mercy Service helicopter is periodically available for transfer of critically injured patients from public rural facilities. The vast majority of patients seen in government hospitals are transported by road ambulance services from peripheral hospitals to the nearest trauma unit.

The only level 1 trauma centre in KZN is Inkosi Albert Luthuli Central Hospital in Durban. Pietermaritzburg is the region's second-largest city, and has two hospitals that receive major trauma referrals from peripheral hospitals up to $200 \mathrm{~km}$ away as part of an informal provincial trauma network. ${ }^{[1]}$ The two hospitals are Edendale Hospital and Grey's Hospital. Edendale Hospital's ED is staffed by emergency medicine specialists and trainees, and Grey's Hospital casualty department is staffed by medical officers from allied specialties. Both hospitals have intensive care units and trauma surgery capability.

\section{Eligibility}

Trauma and burns patients of all ages with emergency tracheal intubation (or an intubation attempt) were eligible for inclusion. These included procedures undertaken in an ED, at a rural referral hospital or on the roadside. Patients declared dead at the scene and those transferred from a hospital ward to an ED for airway intervention and ventilation were excluded.

\section{Data collection}

Data were collected from Edendale Hospital (11 May - 17 July 2016) and Grey's Hospital (23 May - 17 July 2016).

Data were obtained by completion of an anonymised case report file by the practitioner who had performed intubation (or attempted intubation). Practitioners were trained in the completion of the case report file before the study was undertaken. Data recorded included patient demographics, mechanism of injury, indication for intubation, qualification of the intubating practitioner, intubating conditions and anticipated difficulty, equipment (including preprocedural checklist), monitoring and medication used, adverse events and clinical course (Appendix 1). Data fields were adapted from a consensus article, ${ }^{[12]}$ and were not routinely collected in Pietermaritzburg.

To minimise reporting bias, the value of accurate data in order to improve patient outcomes was emphasised during training, and practitioner details were kept anonymous. No individual clinician performance records were analysed. When patients were referred from a rural hospital following intubation or attempted intubation, completed case report files were sent with the patient's referral letter or electronically.

Admissions records at Edendale and Grey's hospitals, and the Pietermaritzburg Metropolitan Trauma Service electronic registry, were examined at the conclusion of the data collection period to identify additional subjects who were admitted with advanced airway interventions, for whom no prospective case report file had been completed.

\section{Outcomes}

The primary outcome of interest was successful tracheal intubation. Secondary outcomes included first-pass success and adverse events occurring at the time of the procedure.

\section{Preintubation checklist}

A preintubation checklist may improve emergency intubation outcomes, ${ }^{[13]}$ and is recommended by recent guidelines. ${ }^{[14]}$ Use of such a list in Pietermaritzburg was recorded in order to ascertain whether there were differences in practice between intubation settings, and whether these were associated with differences in first-pass success and adverse event rates.

\section{Pharmacological agents}

This study recorded anaesthetic medication used to facilitate emergency intubation, and indications for intubation, to assess compliance with guidelines. Currently accepted guidelines state that in traumatic brain injury, patients with a Glasgow Coma Score of $\geq 8$ require intubation before transfer if it can be performed safely. ${ }^{[15]}$ Tracheal intubation requires adequate sedation and muscle relaxation to minimise any increase in intracranial pressure and to reduce the likelihood of aspiration of gastric contents. In patients without cardiac output, the necessity of obtaining an airway supersedes these concerns. ${ }^{[16]}$

\section{Equipment and monitoring}

Current guidelines stipulate that standards of practice and monitoring in prehospital anaesthesia should be comparable to those recommended for in-hospital emergency anaesthesia. Monitoring of end-tidal carbon dioxide is mandatory in prehospital practice, and equipment should include a bougie. ${ }^{[14]}$ Use of this equipment was recorded to investigate whether minimum standards were met.

\section{Definitions}

Interns and grade 1 medical officers were categorised as "junior doctors' and grade 2/grade 3 medical officers, specialists and trainees in emergency medicine, anaesthetics or intensive care 'senior doctors'. Pharmacological adjuncts to intubation were categorised as 'rapidsequence induction' (sedation with neuromuscular blockade) or 'deep sedation' (sedation without neuromuscular blockade). 'Drugless intubation' was defined as intubation where no muscle relaxant or sedative medication was used. This was defined a priori as 'inappropriate' if the patient was not in cardiac arrest, as best practice guidelines state that patients should be appropriately sedated and relaxed. ${ }^{[15]}$

Despite their organisational differences, intubations undertaken in the Edendale ED and the Grey's casualty department were categorised as 'ED' intubations. All referral hospitals outside the Pietermaritzburg conurbation were categorised as 'rural hospitals'.

\section{Study size}

Owing to the observational nature of this study and the lack of available prior data representative of this setting, no sample size calculation was undertaken before commencement. The number of patients recruited was determined by the time period in which the principal investigator was present at the hospital complex.

\section{Data analysis}

Normally distributed data are presented as means and standard deviations (SDs). Categorical data are presented as $n$ (\%), and comparisons between groups were made using $\chi^{2}$ analysis. Missing data were excluded from analysis. A $p$-value of $<0.05$ was considered significant. SPSS v19 (IBM, USA) was used for statistical testing. Multivariate analysis was not performed owing to the small sample size.

\section{Results}

Fifty-seven patients required emergency intubation following trauma and burns during the study period. Case report files were completed for $41 / 57$ of these cases, including 30/30 admissions to Edendale 
Hospital and 11/27 admissions to Grey's Hospital. The remaining 16 patients were identified from retrospective examination of admissions records, but no further data were available.

\section{Patient characteristics}

The 41 subjects had a mean (SD) age of 27 (12) years, and 34/41 were male. The most common mechanism of injury was road traffic collision. Thirty-seven subjects sustained blunt trauma. Burns patients $(n=3)$ suffered injuries affecting 38 - 60\% body surface area, with additional inhalation injury.
Follow-up data were available for $40 / 41$ patients to the point at which they left the Pietermaritzburg trauma system. Of these, 16/40 survived to extubation, 13/40 died in hospital in Pietermaritzburg or were transferred back to the base hospital for palliation, and 11/40 were accepted by the regional neurosurgical facility and transferred to the level 1 trauma centre.

Table 1 displays patient demographics, mechanism of injury, indication for intubation, qualification of intubating practitioner, anticipated difficulty, medication and equipment (including checklist) used, adverse events and clinical course.

\begin{tabular}{|c|c|c|c|c|}
\hline Patient characteristics & $\begin{array}{l}\text { Total, } \\
n(\%)\end{array}$ & $\begin{array}{l}\mathrm{ED}, \\
n(\%)\end{array}$ & $\begin{array}{l}\text { Rural hospital, } \\
n(\%)\end{array}$ & $\begin{array}{l}\text { Roadside, } \\
n(\%)\end{array}$ \\
\hline Total & $41(100)$ & $22(53.7)$ & $8(19.5)$ & $11(26.8)$ \\
\hline Male & $34(82.9)$ & $18(81.8)$ & $7(87.5)$ & $9(81.8)$ \\
\hline \multicolumn{5}{|l|}{ Age (years) } \\
\hline $0-16$ & $9(22.0)$ & $7(31.8)$ & $2(25.0)$ & 0 \\
\hline $17-35$ & $23(56.1)$ & $10(45.5)$ & $5 / 8(62.5)$ & $8(72.7)$ \\
\hline $36-50$ & $8(19.5)$ & $4(18.2)$ & $1(12.5)$ & $3(27.3)$ \\
\hline$\geq 51$ & $1(2.4)$ & $1(4.5)$ & 0 & 0 \\
\hline \multicolumn{5}{|l|}{ Mechanism of injury } \\
\hline Road traffic collision & $21(51.2)$ & $8(36.4)$ & $5(62.5)$ & $8(72.7)$ \\
\hline Assault & $15(36.6)$ & $11(50.0)$ & $2(25.0)$ & $2(18.2)$ \\
\hline Fall & $1(2.4)$ & $1(4.5)$ & 0 & 0 \\
\hline Burns & $3(7.3)$ & $2(9.1)$ & 0 & $1(9.1)$ \\
\hline Unknown (blunt) & $1(2.4)$ & 0 & $1(12.5)$ & 0 \\
\hline \multicolumn{5}{|l|}{ Indication for intubation* } \\
\hline Reduced consciousness & $37(92.5)$ & $19(86.4)$ & $8(100)$ & $10(100)$ \\
\hline Existing/pending airway obstruction & $8(20.0)$ & $3(13.6)$ & 0 & $5(50.0)$ \\
\hline Inadequate ventilation & $14(35.0)$ & $6(27.3)$ & $3(37.5)$ & $5(50.0)$ \\
\hline Cardiac arrest & $1(2.5)$ & 0 & 0 & $1(10.0)$ \\
\hline \multicolumn{5}{|l|}{ First laryngoscopy performed by } \\
\hline Paramedic (government) & $2(4.9)$ & 0 & 0 & $2(18.1)$ \\
\hline Paramedic (private) & $9(22.0)$ & 0 & 0 & $9(81.8)$ \\
\hline Doctor (junior) & $14(34.1)$ & $12(54.5)$ & $2(25.0)$ & 0 \\
\hline Doctor (senior) & $12(29.3)$ & $8(36.4)$ & $4(50.0)$ & 0 \\
\hline Doctor (unknown) & $4(9.8)$ & $2(9.1)$ & $2(25.0)$ & 0 \\
\hline \multicolumn{5}{|l|}{ Anticipation and equipment } \\
\hline Anticipated difficult airway & $12(32.4)$ & $7(35.0)$ & $1(14.3)$ & $4(40.0)$ \\
\hline Preintubation checklist used & $23(57.5)$ & $18(85.7)$ & 0 & $5(45.5)$ \\
\hline Bougie used & $2(5.7)$ & $2(10.5)$ & 0 & 0 \\
\hline Video laryngoscope used & $16(39.0)$ & $16(72.7)$ & 0 & 0 \\
\hline End-tidal $\mathrm{CO}_{2}$ monitoring used & $25(61.0)$ & $18(81.8)$ & 0 & $7(63.6)$ \\
\hline \multicolumn{5}{|l|}{ Pre-existing airway debris } \\
\hline None & $15(40.5)$ & $11(55.0)$ & $2(28.6)$ & $2(20.0)$ \\
\hline Mild & $13(35.1)$ & $8(40.0)$ & $2(28.6)$ & $3(30.0)$ \\
\hline Moderate & $6(16.2)$ & 0 & $2(28.6)$ & $4(40.0)$ \\
\hline Severe & $3(8.1)$ & $1(5.0)$ & $1(14.3)$ & $1(10.0)$ \\
\hline \multicolumn{5}{|l|}{ Pharmacological technique } \\
\hline Rapid-sequence induction & $27(65.9)$ & $18(81.8)$ & $4(50.0)$ & $5(45.5)$ \\
\hline Deep sedation & $9(22.0)$ & $3(13.6)$ & $1(12.5)$ & $5(45.5)$ \\
\hline None & $5(12.2)$ & $1(4.5)$ & $3(37.5)$ & $1(9.1)$ \\
\hline Inappropriate drugless intubation & $4(9.8)$ & $1(4.5)$ & $3(37.5)$ & 0 \\
\hline \multicolumn{5}{|l|}{ Clinical course } \\
\hline Survived to extubation & $16(40.0)$ & $10(47.6)$ & $2(25.0)$ & $4(36.4)$ \\
\hline Accepted by neurosurgery & $11(27.5)$ & $8(38.1)$ & $2(25.0)$ & $1(9.1)$ \\
\hline Died in hospital & $13(32.5)$ & $3(14.3)$ & $4(50.0)$ & $6(54.5)$ \\
\hline
\end{tabular}




\section{Outcomes}

All included patients were successfully intubated; this was achieved on first pass for 28/39 patients for whom data were available. Successful first-pass intubation was more likely in an ED than in rural hospitals $(19 / 22$ v. $2 / 7 ; p=0.003)$. Identified adverse events due to intubation were $1 / 22$ (ED), 5/8 (rural hospitals) and 2/9 (roadside). Table 2 displays first-pass success and adverse event rates, Table 3 compares outcomes with and without the use of a preintubation checklist, and Table 4 details the nature of recorded adverse events.

\section{Preintubation checklist}

There was wide variation in use of the preintubation checklist, as illustrated in Table 1. Overall, its use was associated with higher first-pass success rates $(p=0.001)$ and fewer adverse events $(p<0.001)$, as shown in Table 3. No rural hospital intubations were done using a checklist.

\section{Pharmacological agents}

Medication used for intubation is displayed in Table 1. Five patients were intubated with neither sedative nor muscle-relaxing medication, and $4 / 5$ of these procedures were deemed inappropriate owing to absence of cardiac arrest. Inappropriate drugless intubation was more prevalent in rural hospitals than EDs $(p=0.019)$, as shown in Table 2.

\section{Equipment and monitoring}

There was heterogeneity in practice between locations in the use of introducers, video laryngoscopes and end-tidal carbon dioxide monitoring, as illustrated in Table 1. In particular, none of these modalities were employed in rural hospitals.

\section{Discussion}

We found that intubation practice, first-pass success rates and associated complication rates in trauma and burns patients were variable between settings. Rural hospitals recorded a particularly low first-pass success rate and a considerable adverse event rate. Use of a preintubation checklist was associated with lower intubation-related morbidity in the ED and on the roadside, but a checklist was not used in the rural hospital setting. Minimum standards for prehospital monitoring of anaesthesia ${ }^{[14]}$ were typically unmet.

\section{Preintubation checklist}

Recent guidelines emphasise the usefulness of a preinduction checklist in both prehospital and ED environments. ${ }^{[14]}$ Although causality cannot be established from an observational study, particularly of this small magnitude, this study found the use of a formal checklist to be associated with a higher rate of first-pass success. This is consistent with current literature internationally. ${ }^{[13]}$

Edendale Hospital's ED emergency intubation checklist is an A4 laminated sheet attached to each airway trolley and covering planning, medication, patient preparation and equipment required. It also depicts the Difficult Airway Society's advanced airway algorithm, ${ }^{[17]}$ including rescue techniques for 'can't intubate, can't ventilate' scenarios.

It is unlikely that there is sufficient ethical equipoise to justify a randomised controlled trial. However, a simplified algorithm has been proposed for the rural hospital setting. ${ }^{[9]}$ This inexpensive intervention could be implemented readily and may be particularly beneficial in rural hospitals, and its value could be tested through pre- and post-intervention quality improvement data on a siteby-site basis. The data generated at each participating site could be pooled regionally to increase power, and then used to audit SA practice against international consensus guidelines with a view to standardising care. Although this would require considerable cross-

\begin{tabular}{|c|c|c|c|}
\hline Outcome by location & $\begin{array}{l}\text { Checklist, } \\
n(\%)\end{array}$ & $\begin{array}{l}\text { No checklist, } \\
n(\%)\end{array}$ & $p$-value \\
\hline \multicolumn{4}{|l|}{ First-pass success } \\
\hline Overall & $21(91.3)$ & $6(40.0)$ & $0.001^{*}$ \\
\hline $\mathrm{ED}$ & $16(88.9)$ & $2(66.7)$ & 0.285 \\
\hline Rural hospital & + & $2(28.6)$ & $\dagger$ \\
\hline Roadside & $5(100)$ & $2(40.0)$ & $0.038^{*}$ \\
\hline \multicolumn{4}{|l|}{$\geq 1$ adverse event } \\
\hline Overall & 0 & $7(43.8)$ & $<0.001^{*}$ \\
\hline $\mathrm{ED}$ & 0 & $1(33.3)$ & $0.010^{*}$ \\
\hline Rural hospital & $\dagger$ & $4(57.1)$ & $\dagger$ \\
\hline Roadside & 0 & $2(33.3)$ & 0.154 \\
\hline \multicolumn{4}{|c|}{$\begin{array}{l}\text { Missing data are excluded from reported percentages. } \\
\text { ED = emergency department. } \\
\\
\text { t } p<0.05 \text { considered statistically significant. } \\
\text { 'No rural hospitals used checklist; statistical test not performed. }\end{array}$} \\
\hline
\end{tabular}

Table 4. Adverse events at time of intubation, according to location $^{*}$

\begin{tabular}{|c|c|c|c|}
\hline Complication & $\begin{array}{l}\mathrm{ED}, \\
n(\%)\end{array}$ & $\begin{array}{l}\text { Rural } \\
\text { hospital, } \\
n(\%)\end{array}$ & $\begin{array}{l}\text { Roadside, } \\
n(\%)\end{array}$ \\
\hline Oesopheageal tube (identified) & 0 & $2(25.0)$ & 0 \\
\hline Bronchial tube (unidentified) & 0 & $2(25.0)$ & 0 \\
\hline Bronchial tube (identified) & $1(4.5)$ & 0 & 0 \\
\hline Inappropriate cuff pressure & 0 & 0 & $1(11.1)$ \\
\hline Vomiting, aspiration or both & 0 & $3(37.5)$ & $1(11.1)$ \\
\hline Hypoxaemia $\left(\mathrm{SpO}_{2}<92 \%\right)$ & 0 & $2(25.0)$ & 0 \\
\hline Pneumothorax & 0 & $1(12.5)$ & 0 \\
\hline Dental trauma & 0 & $2(25.0)$ & 0 \\
\hline
\end{tabular}

Table 2. Patient outcomes according to location

\begin{tabular}{|c|c|c|c|c|c|}
\hline \multirow[b]{2}{*}{ Outcome } & \multicolumn{3}{|c|}{ Location } & \multicolumn{2}{|c|}{ Comparisons } \\
\hline & $\begin{array}{l}\mathrm{ED}, \\
n(\%)\end{array}$ & $\begin{array}{l}\text { Rural hospital, } \\
n(\%)\end{array}$ & $\begin{array}{l}\text { Roadside, } \\
n(\%)\end{array}$ & $\begin{array}{l}p \text {-value, } \\
\text { ED v. rural }\end{array}$ & $\begin{array}{l}p \text {-value, } \\
\text { ED v. roadside }\end{array}$ \\
\hline Inappropriate drugless intubation & $1(4.5)$ & $3(37.5)$ & 0 & $0.019^{*}$ & 0.473 \\
\hline First-pass success & $19(86.3)$ & $2(28.6)$ & $7(70.0)$ & $0.003^{*}$ & 0.272 \\
\hline$\geq 1$ adverse event & $1(4.5)$ & $5(62.5)$ & $2(22.2)$ & $<0.001^{*}$ & 0.199 \\
\hline
\end{tabular}


party co-operation across hospital and prehospital providers, the current study shows that it is achievable.

\section{Pharmacological agents}

Adherence to best pharmacological practice in emergency intubation was variable between locations. In order to reduce intubationrelated morbidity, particular attention to this issue may be warranted.

The stimulation caused by tracheal intubation without pharmacological adjuncts can increase intracranial pressure, increasing morbidity in head-injured patients with a reduced level of consciousness. Stimulation of the gag reflex may also elicit vomiting and lead to aspiration in this setting. This study found that a neuromuscular blocking agent was used in only half of rural hospital intubations. In some rural facilities, suxamethonium is kept in operating theatres but not in the casualty department. Hospital policies may stipulate a doctor's presence and signature before the drug is removed from theatres. In an emergency situation with an urgent need to secure an airway and limited medical staffing, obtaining suxamethonium in a timely manner may be impractical owing to these barriers.

In rural hospital casualty departments, $3 / 8$ intubations involved no sedative or muscle-relaxing medication. All took place in the context of traumatic brain injury in the absence of cardiac arrest. Plausible explanations include inability to access drugs in a timely manner, or inability to administer drugs owing to lack of intravenous access prior to intubation. The introduction of a tracheal tube without the use of pharmacological adjuncts puts these patients at considerable risk of harm, both physiological and anatomical, and raises questions about the balance of risks $\mathrm{v}$. benefits of advanced airway interventions in this patient group.

To reduce these risks, appropriate drugs should be readily accessible in the emergency setting prior to intubation, and the use of supraglottic devices or simple airway manoeuvres may be more appropriate in some cases.

A standardised rapid-sequence induction protocol has been demonstrated to improve outcomes elsewhere, ${ }^{[18]}$ and may be useful in rural KZN. Intraosseous administration of anaesthesia is effective in rapid-sequence induction, ${ }^{[19]}$ and may also warrant consideration.

\section{Equipment and monitoring}

International guidelines state that difficult intubation should be anticipated in the prehospital setting, and recommend routine use of intubating bougies. ${ }^{[14]}$ Bougies were rarely used in emergency intubation of trauma and burns patients in Pietermaritzburg and its drainage area. The use of a metal stylet as an adjunct was not assessed in this study, but some providers reported that they would only employ an introducer after a failed first attempt or in a difficult airway situation. International research suggests poor diagnostic accuracy in predicting difficult tracheal intubation, ${ }^{[20]}$ and this also appears true in KZN: only $1 / 7$ of rural hospital intubations were anticipated to be in difficult airways, yet the first-pass success rate was just 2/7. Encouraging the routine use of an introducer in all emergency intubations in trauma and burns patients, irrespective of anticipated difficulty, may improve the likelihood of first-pass success and reduce intubation-related complications.

End-tidal carbon dioxide monitoring was used in 18/22 ED intubations, compared with just 7/19 of prehospital cases. As such, recommended minimum standards of emergency anaesthetic monitoring ${ }^{[21]}$ were frequently unmet.

\section{Study limitations}

This study is limited by its small sample, the self-administered nature of case report files, potential heterogeneity of subjects and injury severity from different sites, and incomplete follow-up. Meaningful multivariate statistical analyses could not be performed owing to sample size. For logistical reasons, patients accepted by the regional neurosurgical centre could not be followed up once they left Pietermaritzburg. A high turnover of medical staff in the Grey's Hospital casualty department, combined with the absence of comprehensive electronic patient tracking and record-keeping systems across KZN, presented challenges to data collection. The background rate of emergency airway management in trauma across KZN is not ascertained, although regional data (e.g. Pietermaritzburg) are potentially deducible from local registries such as the Pietermaritzburg Metropolitan Trauma Service database.

Despite reassurances during briefing, the commercial nature of private ambulance services and the impossibility of data verification make the current study at risk of potential over-reporting of success and under-reporting of adverse events. Nonetheless, the high recorded incidence of adverse events, even in such a small study, highlights the opportunity for improving the safety of advanced airway intervention in these patients.

Possible adverse events following attempted tracheal intubation include misplaced tube, inappropriate cuff pressure, pneumothorax, vomiting, aspiration, hypoxaemia, hypotension, bradycardia and cardiac arrest; laryngoscopy also carries the risk of dental trauma. ${ }^{[12,22]}$ Intubation-related morbidity cannot be accurately quantified through a study of this nature, as it may not be possible to establish with certainty whether events recognised at the time of intubation (e.g. dental trauma) were attributable to laryngoscopy and intubation or caused as a direct result of injury mechanism, and episodes such as hypoxaemia occurring at the time cannot be verified retrospectively.

\section{External validity}

The trauma system in KZN is typical of practice across SA. It is possible that data from other developing trauma systems of similar scale and logistical framework might be in keeping with our findings. Our recommendations may serve to improve patient care in comparable situations.

\section{Conclusions}

Clinical heterogeneity exists during emergency intubation for trauma and burns patients in the ED, prehospital and rural settings of KZN, SA. The use of a preprocedural checklist is associated with improved outcomes and may improve quality of care. Standardised rapid-sequence induction protocols, routine use of introducers and end-tidal carbon dioxide monitoring, and increased availability of intraosseous devices may also yield improvements in patient outcomes. These recommendations are in line with recently updated international guidelines, which also suggest monitoring of key performance indicators. Further research in this setting is both feasible and warranted.

Acknowledgements. We are grateful to the Pietermaritzburg paramedic crews and medical staff at Edendale Hospital, Grey's Hospital and peripheral hospitals for their engagement with this project. We appreciate the support of hospital management for permitting data collection on their premises, and of the Pietermaritzburg Metropolitan Trauma Service for access to their trauma database. CTL would like to thank the Sir Arthur Thomson Charitable Trust, Association of Anaesthetists of Great 
Britain and Ireland, British Medical and Dental Students' Trust and Royal College of Anaesthetists for their support of this study through their elective bursary award schemes.

Author contributions. All authors contributed to the conception and design of the study. CTL, JB and ACI collected data. CTL, DNN and NC analysed the data, interpreted results and prepared the manuscript. All authors critically revised and approved the manuscript and are accountable for its integrity.

\section{Funding. None.}

Conflicts of interest. None.

1. Fevang E, Lockey D, Thompson J, et al.; Torpo Research Collaboration. The top five research priorities in physician-provided pre-hospital critical care: A consensus report from a European research collaboration. Scand J Trauma Resusc Emerg Med 2011;19:57. https://doi.org/10.1186/1757-7241-19-57

2. Lecky F, Bryden D, Little R, et al. Emergency intubation for acutely ill and injured patients. Cochrane Database Syst Rev 2008, Issue 2. Art. No.: CD001429. https://doi.org/10.1002/14651858.cd001429. pub2

3. Lockey DJ, Healey B, Crewdson K, et al. Advanced airway management is necessary in pre-hospital trauma patients. Br J Anaesth 2015;114(4):657-662. https://doi.org/10.1093/bja/aeu412

4. Prekker ME, Kwok H, Shin J, et al. The process of pre-hospital airway management: Challenges and solutions during paramedic endotracheal intubation. Crit Care Med 2014;42(6):1372-1378. https://doi org/10.1097/ccm.0000000000000213 4. Lossius HM, Røislien J, Lockey DJ. Patient safety in pre-hospital emergency tracheal intubation:
A comprehensive meta-analysis of the intubation success rates of EMS providers. Crit Care 2012;16:R24. https://doi.org/10.1186/cc11189

5. Lutge E, Moodley N, Tefera A, et al. A hospital based surveillance system to assess the burden of trauma in KwaZulu-Natal Province South Africa. Injury 2016;47(1):135-140. https://doi.org/10.1016/j. injury.2015.08.020

6. Hardcastle TC, Finlayson M, van Heerden M, et al. The pre-hospital burden of disease due to trauma in KwaZulu-Natal: The need for Afrocentric trauma systems. World J Surg 2013;37(7):1513-1525. https://

7. Stein C, Botha M, Kramer E, et al. Position statement: Pre-hospital rapid sequence intubation. S Afr Med J 2011;101(3):163. https://doi.org/10.7196/SAMJ.4230

8. Berry M, Wood D. Simplifying trauma airway management in South African rural hospitals. S Afr Med J 2014;104(9):604-606. https://doi.org/10.7196/SAMJ.8064
9. Biccard BM, Alphonsus CS, Bishop DG, et al. National priorities for perioperative research in South Africa. S Afr Med J 2016;106(5):58-59. https://doi.org/10.7196/SAMJ.2016.v106i5.10269

0. Hardcastle TC, Reeds MG, Muckart DJ. Utilisation of a level 1 trauma centre in KwaZulu-Natal: . Hardcastle TC, Reeds MG, Muckart DJ. Utilisation of a level 1 trauma centre in KwaZulu-Natal:
Appropriateness of referral determines trauma patient access. World J Surg 2013;37(7):1544-1549. Appropriateness of referral determines tram

11. Sollid SJ, Lockey D, Lossius HM; Pre-hospital Advanced Airway Management Expert Group. A consensus-based template for uniform reporting of data from pre-hospital advanced airway management. Scand J Trauma Resusc Emerg Med 2009;17:58. https://doi.org/10.1186/1757-7241-1758

12. Smith KA, High K, Collins SP, et al. A preprocedural checklist improves the safety of emergency department intubation of trauma patients. Acad Emerg Med 2015;22(8):989-992. https://doi. org/10.1111/acem.12717

13. Lockey DJ, Crewdson K, Davies G, et al. AAGBI: Safer pre-hospital anaesthesia 2017: Association of Anaesthetists of Great Britain and Ireland. Anaesthesia 2017;72(3):379-390. https://doi.org/10.1111/ anae. 13779

14. Farling PA, Andrews PJD, Cruickshank S, et al. Association of Anaesthetists of Great Britain and Ireland: Recommendations for the safe transfer of patients with brain injury. 2006. https://www.aagbi. Ireland: Recommendations for the safe transfer of patients $\mathrm{org} / \mathrm{sites} / \mathrm{default} / \mathrm{flies} / \mathrm{braininjury}$.pdf (accessed 2 July 2018).

15. American College of Surgeons. Advanced Trauma Life Support (ATLS): The Ninth Edition. Chicago: ACS, 2012.

16. Frerk C, Mitchell VS, McNarry AF, et al.; Difficult Airway Society intubation guidelines working group. Difficult Airway Society 2015 guidelines for management of unanticipated difficult intubation in adults. Br J Anaesth 2015;115(6):827-848. https://doi.org/10.1093/bja/aev37

17. Ballow SL, Kaups KL, Anderson S, et al. A standardized rapid sequence intubation protocol facilitates airway management in critically injured patients. J Trauma Acute Care Surg 2012;73(6):1401-1405. https://doi.org/10.1097/ta.0b013e318270dcf5

18. Barnard EB, Moy RJ, Kehoe AD, et al. Rapid sequence induction of anaesthesia via the intraosseous route: A prospective observational study. Emerg Med J 2015;32(6):449-452. https://doi.org/10.1136/ emermed-2014-203740

19. Nørskov AK, Rosenstock CV, Wetterslev J, et al. Diagnostic accuracy of anaesthesiologists' prediction of difficult airway management in daily clinical practice: A cohort study of 188064 patients registered in the Danish Anaesthesia Database. Anaesthesia 2015;70(3):272-281. https://doi.org/10.1111/ anae. 12955

20. Checketts MR, Alladi R, Ferguson K, et al.; Association of Anaesthetists of Great Britain and Ireland. Recommendations for standards of monitoring during anaesthesia and recovery 2015. Anaesthesia 2016;71(1):85-93. https://doi.org/10.1111/anae.13316

21. Hardcastle TC, Faurie M, Muckart DJJ. Endotracheal tube cuff pressures and tube position in critically injured patients on arrival at a referral centre: Avoidable harm? Afr J Emerg Med 2016;6(1):24-29. https://doi.org/10.1016/j.afjem.2015.09.002

Accepted 1 March 2018 


\section{Appendix 1. Intubation data collection form}

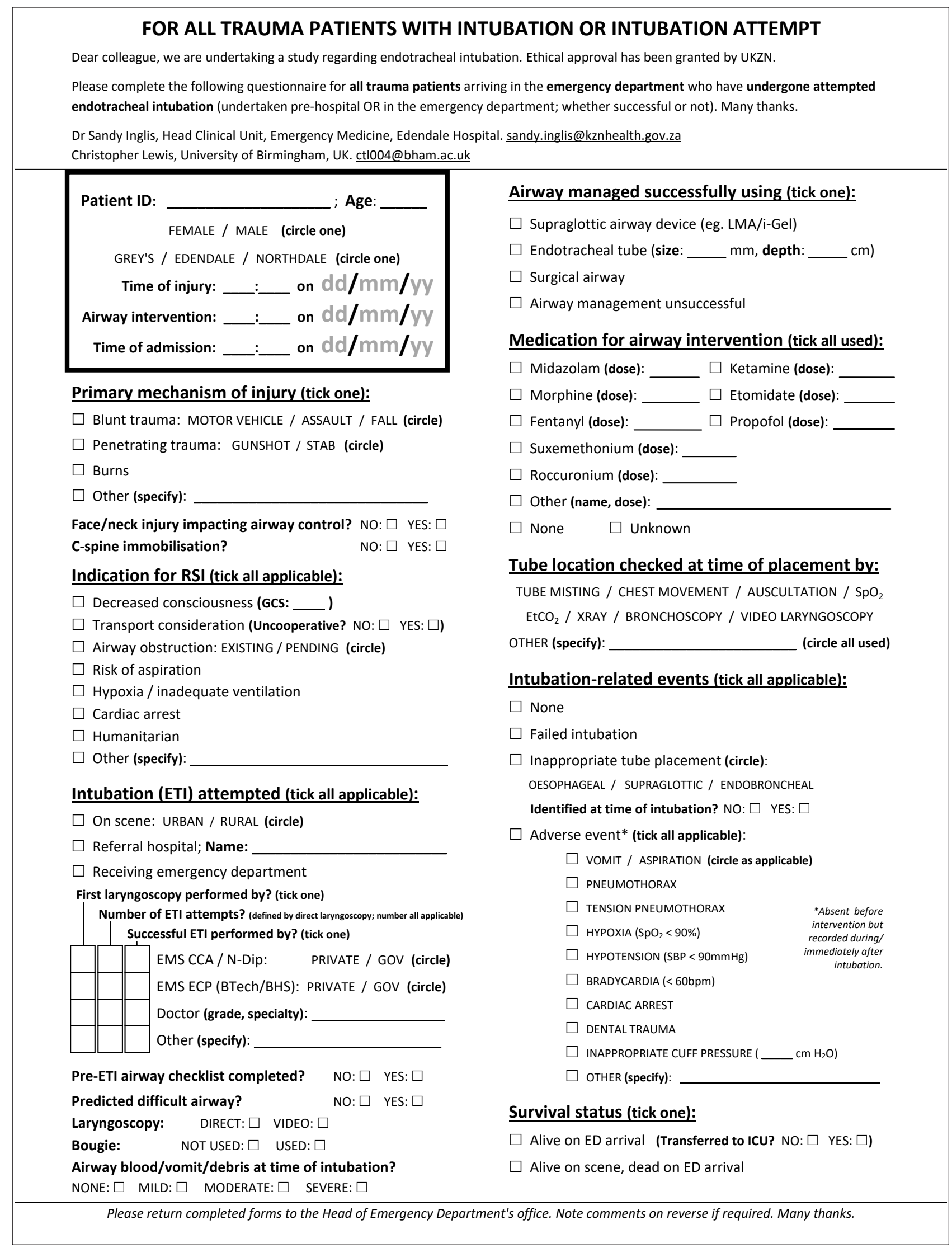

